\title{
Anisotropic magnetocaloric effect and critical behavior in $\mathrm{CrCl}_{3}$
}

\author{
Yu Liu and C. Petrovic \\ Condensed Matter Physics and Materials Science Department, \\ Brookhaven National Laboratory, Upton, New York 11973, USA
}

(Dated: July 15, 2020)

\begin{abstract}
We report anisotropic magnetocaloric effect and magnetic critical behavior in van der Waals crystal $\mathrm{CrCl}_{3}$. The maximum magnetic entropy change $-\Delta S_{M}^{\max } \sim 14.6 \mathrm{~J} \mathrm{~kg}^{-1} \mathrm{~K}^{-1}$ and the relative cooling power $R C P \sim 340.3 \mathrm{~J} \mathrm{~kg}^{-1}$ near $T_{c}$ with a magnetic field change of $5 \mathrm{~T}$ are much larger when compared to $\mathrm{CrI}_{3}$ or $\mathrm{CrBr}_{3}$. The rescaled $\Delta S_{M}(T, H)$ curves collapse onto a universal curve, confirming the second order ferromagnetic transition. Further critical behavior analysis around $T_{c}$ presents a set of critical exponents $\beta=0.28(1)$ with $T_{c}=19.4(2) \mathrm{K}, \gamma=0.89(1)$ with $T_{c}=18.95(8)$ $\mathrm{K}$, and $\delta=4.6(1)$ at $T_{c}=19 \mathrm{~K}$, which are close to those of theoretical tricritical mean field model.
\end{abstract}

\section{INTRODUCTION}

Chromium trihalides $\mathrm{CrX}_{3}(\mathrm{X}=\mathrm{Cl}, \mathrm{Br}$, and $\mathrm{I})$ have triggered a renewed interest since the recent discovery of intrinsic two-dimensional (2D) magnetism in monolayer

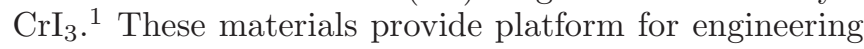
of novel spintronic devices and studies of $2 \mathrm{D}$ spin order. The microscopic mechanism for properties of interest stems from the layered antiferromagnetic (AFM) ground state and the low critical magnetic fields required for a ferromagnetic (FM) phase transition (6 $\mathrm{kOe}$ and 11 kOe for bilayer $\mathrm{CrI}_{3}$ and $\mathrm{CrCl}_{3}$, respectively) $\underline{\underline{1}} \underline{\underline{\underline{8}}}$

Bulk $\mathrm{CrI}_{3}$ and $\mathrm{CrBr}_{3}$ are $\mathrm{FM}$ with the Curie temperature $\left(T_{c}\right)$ of $61 \mathrm{~K}$ and $37 \mathrm{~K}, \underline{\underline{9}-11}$ respectively, whereas $\mathrm{CrCl}_{3}$ is AFM with the Néel temperature $\left(T_{N}\right)$ of 16.8 $\mathrm{K} . \stackrel{12}{2}$ Bulk $\mathrm{CrX}_{3}$ crystalize in the layered $\mathrm{BiI}_{3}$-type structure, space group $\mathrm{R} \overline{3}$. The edge-shared $\mathrm{CrX}_{6}$ octahedra form a $2 \mathrm{D}$ honeycomb layers of $\mathrm{Cr}$ ions. These sandwiched $\mathrm{X}-\mathrm{Cr}-\mathrm{X}$ slabs are stacked along the $c$ axis and are held by weak van der Waals (vdW) interactions. The different radii of $\mathrm{X}$ alter the in-plane nearest-neighbor $\mathrm{Cr}-\mathrm{Cr}$ distance and the vdW gap between X-Cr-X slabs. From $\mathrm{I}$ to $\mathrm{Br}$ to $\mathrm{Cl}$, the $\mathrm{X}-\mathrm{Cr}-\mathrm{X}$ bonding is less covalent, weakening superexchange interactions and lowering ordering temperature $\stackrel{2}{2}$ For $\mathrm{CrCl}_{3}$, neutron scattering and NMR experiments show a three-dimensional (3D) AFM magnetic structure at low temperature, consisting of alternating FM sheets of spins aligned within the Cr planes $\underline{\underline{12}}$ The transition temperature of $17 \mathrm{~K}$ was characterized by heat capacity measurement, $, 13,14$ and recently updated with two heat capacity peaks at $T_{N}=14 \mathrm{~K}$ and $T_{C}$ $=17 \mathrm{~K} \stackrel{2}{2}$ Faraday rotation, magnetization, and neutron diffraction measurements show that the ordered state of $\mathrm{CrCl}_{3}$ has a weak magnetic anisotropy, and fields of only a few $\mathrm{kOe}$ are required to fully polarize the magnetization in- or out-of the Cr plane ${ }^{15-17}$ The magnetocaloric effect (MCE) of vdW magnets can give additional insight into the magnetic properties, and it can also be used to assess magnetic refrigeration potential. Bulk $\mathrm{CrI}_{3}$ exhibits anisotropic magnetic entropy change $\left(-\Delta S_{M}^{\max }\right)$ with values of 4.24 and $2.68 \mathrm{~J} \mathrm{~kg}^{-1} \mathrm{~K}^{-1}$ at $50 \mathrm{kOe}$ for outof-plane and in-plane fields, respectively 18 The value of $-\Delta S_{M}^{\max }$ is about $7.2 \mathrm{~J} \mathrm{~kg}^{-1} \mathrm{~K}^{-1}$ at $50 \mathrm{kOe}$ for $\mathrm{CrBr}_{3} \stackrel{19}{\underline{19}}$
Typical 3D magnetic critical behavior is present in $\mathrm{CrI}_{3}$ crystals $\stackrel{20,21}{2}$ However, the magnetocaloric properties and critical behavior of $\mathrm{CrCl}_{3}$ are still unknown.

In the present work we investigate the unusual twostep magnetic ordering process of bulk $\mathrm{CrCl}_{3}$ single crystals by detailed measurements of $\mathrm{dc}$ and ac magnetization. The AFM ground state below $T_{N}=14.4 \mathrm{~K}$ is observed, and an intermediate FM before transition into PM state on heating is also confirmed. The values of $-\Delta S_{M}^{\max } \sim 14.6 \mathrm{~J} \mathrm{~kg}^{-1} \mathrm{~K}^{-1}$ and the relative cooling power $(R C P) \sim 340.3 \mathrm{~J} \mathrm{~kg}^{-1}$ near the PM-FM phase transition with field change of $5 \mathrm{~T}$, indicating that $\mathrm{CrCl}_{3}$ would be a promising candidate material for cryomagnetic refrigeration. The scaling analysis of $\Delta S_{M}(T, H)$ reveals that the PM-FM phase transition is of secondorder in nature. A set of critical exponents is further estimated, $\beta=0.26(1), \gamma=0.86(1)$, and $\delta=4.6(1)$, indicating that the PM-FM transition at $T_{c} \sim 19 \mathrm{~K}$ of bulk $\mathrm{CrCl}_{3}$ is situated close to a $3 \mathrm{D}$ to $2 \mathrm{D}$ critical point.

\section{EXPERIMENTAL DETAILS}

$\mathrm{CrCl}_{3}$ single crystals were grown by recrystalizing commercial $\mathrm{CrCl}_{3}$ (Alpha Aesar, 99.9\%) polycrystal using the chemical vapor transport (CVT) method. The starting material was sealed in a quartz tube in vacuum and then placed inside a two-zone horizontal tube furnace with source and growth temperatures up to $650{ }^{\circ} \mathrm{C}$ and 550 ${ }^{\circ} \mathrm{C}$, respectively, for 7 days. Large, thin, violet-colored, transparent plate-like single crystals with lateral dimensions up to several millimeters can be obtained. The x-ray diffraction (XRD) data were taken with $\mathrm{Cu} \mathrm{K}_{\alpha}$ $(\lambda=0.15418 \mathrm{~nm})$ radiation of Rigaku Miniflex powder diffractometer. The magnetic properties were characterized by the quantum design magnetic property measurement system (MPMS-XL5). The dc magnetization was measured at various magnetic fields from 5 Oe to $50 \mathrm{kOe}$. The isothermals were measured up to $50 \mathrm{kOe}$ in $\Delta T=1$ $\mathrm{K}$ intervals. 

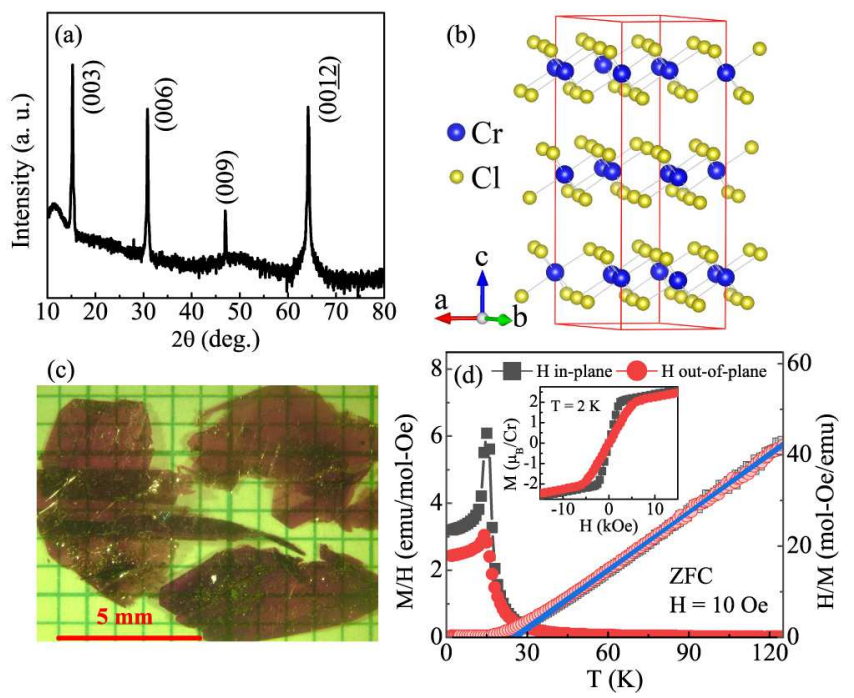

FIG. 1. (Color online). (a) X-ray diffraction (XRD) pattern in $\log$ scale of $\mathrm{CrCl}_{3}$ single crystal. $\mathrm{BiI}_{3}$-type structure (b) at low temperature and representative single crystals (c). (d) Temperature dependence of zero field cooling (ZFC) normalized magnetization (left axis) $M / H$ and (right axis) $H / M$ of $\mathrm{CrCl}_{3}$ at $\mathrm{H}=10$ Oe applied along in-plane and out-of-plane directions. Inset shows the field dependence of magnetization $M(H)$ at $2 \mathrm{~K}$.

\section{RESULTS AND DISCUSSIONS}

The XRD pattern can be well indexed by the indices of (00l) plane, indicating that the crystal surface is parallel to the $a b$ plane [Figs. $1(\mathrm{a}-\mathrm{c})]$. The in-plane and out-ofplane directions are parallel and perpendicular to the $a b$ plane, respectively. It should be noted that $\mathrm{CrX}_{3}$ share similar structural transitions from the low-temperature rhombohedral to high-temperature monoclinic symmetry with $\mathrm{CrX}_{3}: 210 \mathrm{~K}$ for $\mathrm{CrI}_{3}, 420 \mathrm{~K}$ for $\mathrm{CrBr}_{3}$, and 230 $\mathrm{K}$ for $\mathrm{CrCl}_{3}$, respectively ${ }^{22}$ The interlayer spacing $d=$ $5.8 \AA$ is calculated from the peak positions using Bragg's law $n \lambda=2 d \sin \theta$, consistent with the previously reported value 23 Here we focus on the low-temperature magnetic properties of $\mathrm{CrCl}_{3}$. Figure $1(\mathrm{~d})$ shows the temperature dependence of normalized magnetization $M / H$ at $H=$ 10 Oe applied parallel and perpendicular to the $a b$ plane as well as the inverse values with a temperature step of $1 \mathrm{~K}$. Since there is no significant difference of the zerofield-cooling (ZFC) and field-cooling (FC) data only the ZFC curves are presented. The $M / H$ curves show a peak near $15 \mathrm{~K}$ for both field directions. The downturn below $15 \mathrm{~K}$ points to the reported AFM ground state, while the rapid increase just above it hints towards a possible FM intermediate state. The high temperature data can be fitted by the Curie-Weiss law, giving the effective magnetic moment of $4.32(2) / 4.37(2) \mu_{B} / \mathrm{Cr}$, somewhat larger than the expected value $3.87 \mu_{B}$ for spin-only $\mathrm{Cr}^{3+}$ ion, and the Weiss temperature of $26.1(3) / 24.5(2) \mathrm{K}$ for in-plane/out-of-plane field. The positive Weiss temper-
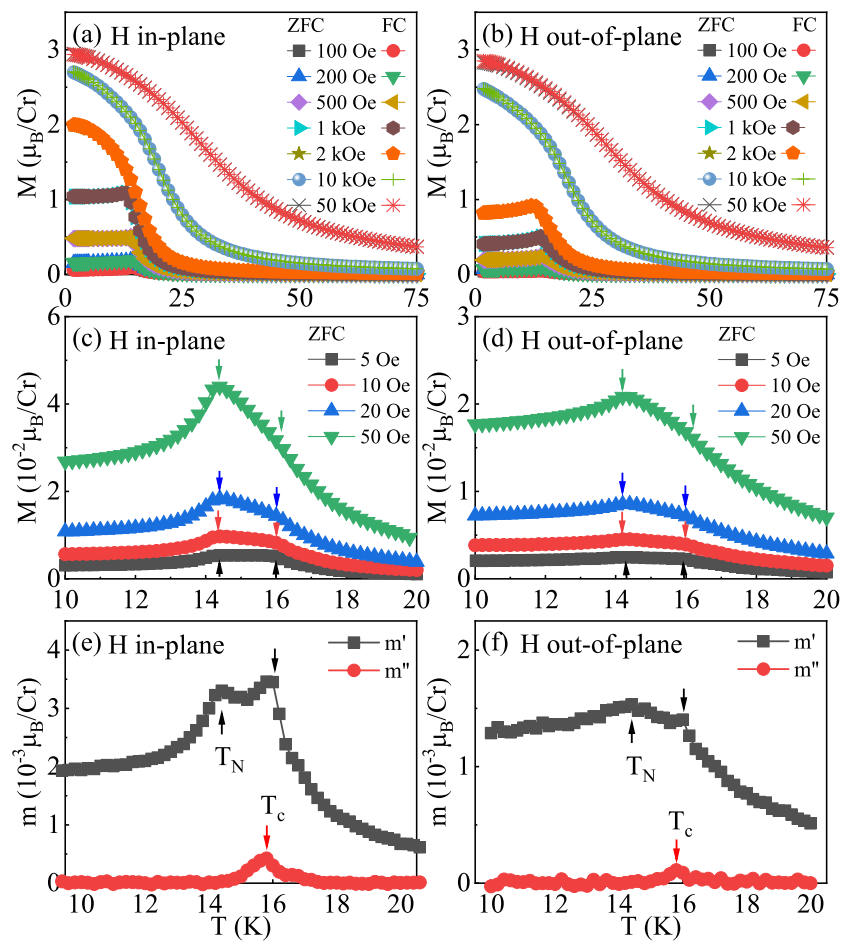

FIG. 2. (Color online). Temperature dependence of zero field cooling (ZFC) and field cooling (FC) dc magnetization $M(T, H)$ of $\mathrm{CrCl}_{3}$ measured at the indicated (a,c) in-plane and (b,d) out-of-plane magnetic fields. Temperature dependence of ac susceptibility real part $m^{\prime}(T)$ and imaginary part $m^{\prime \prime}(T)$ measured with oscillated ac field of 3.8 Oe and frequency of $499 \mathrm{~Hz}$ applied (e) in-plane and (f) out-of-plane.

ature indicates that the FM interactions dominate the magnetic behavior in the paramagnetic (PM) state. The isothermal magnetization of $\mathrm{CrCl}_{3}$ at $2 \mathrm{~K}$ for both field directions shows negligible hysteresis, as shown in inset of Fig. 1(d). The in-plane data change to a weak field-dependence at a smaller field compared to the outof-plane data indicating in-plane easy axis and a weak anisotropy. The linear increase at low fields shows characteristic of the behavior expected for polarizing an antiferromagnet with weak anisotropy.

The temperature dependence of the magnetic moment per Cr measured at various fields near the magnetic transition is shown in Figs. 2(a) and 2(b). At $2 \mathrm{~K}$ and 50 $\mathrm{kOe}$ a moment of $3.0 \mu_{B} / \mathrm{Cr}$ is obtained, as expected for $\mathrm{Cr}^{3+}$ with $\mathrm{S}=3 / 2$. The curves are very similar to those reported in Ref. 2. Kuhlow reported similar behavior in Faraday-rotation measurements, 15 which was interpreted as 2D FM order within the layers developing first, with interlayer long-range AFM order setting in at lower temperature. In order to characterize this two-step magnetic ordering process in $\mathrm{CrCl}_{3}$, we further present the $M(T)$ data at low fields below 100 Oe with a temperature step of $0.2 \mathrm{~K}$ [Figs. 2(c) and 2(d)]. Two distinct peaks in $14.4 \mathrm{~K}$ and $16.0 \mathrm{~K}$ are clearly observed in low-field dc magnetization, as well as in the real part $m^{\prime}$ of ac mag- 

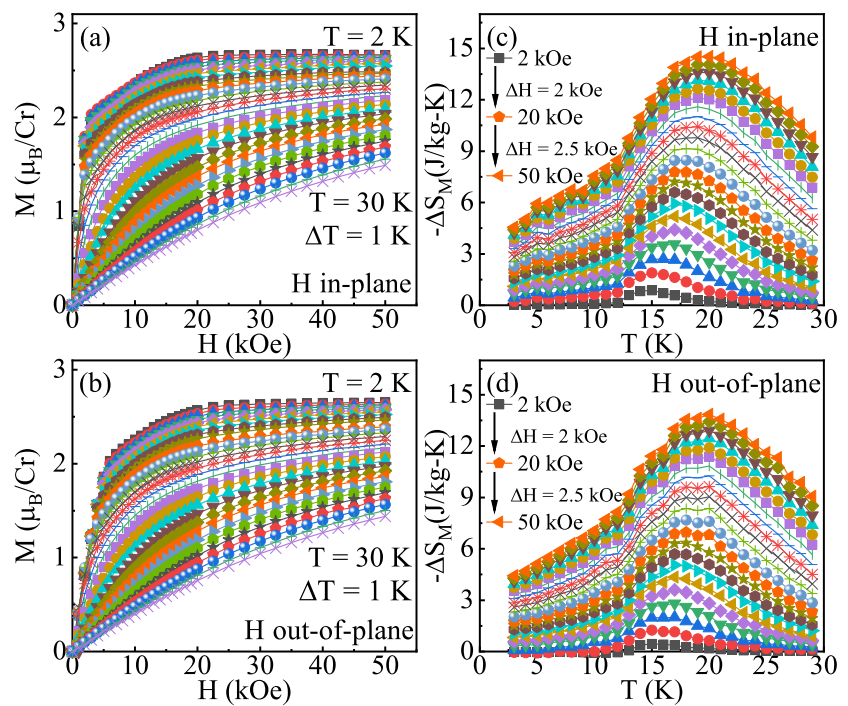

FIG. 3. (Color online). Representative magnetization isothermals at various temperatures around $T_{c}$ for (a) in-plane and (b) out-of-plane magnetic fields. Temperature dependence of the derived magnetic entropy change $-\Delta S_{M}$ at various (c) in-plane and (d) out-of-plane magnetic fields.

netization [Figs. 2(e) and 2(f)] . The imaginary part $m^{\prime \prime}$ feature a peak anomaly at $16.0 \mathrm{~K}$ but not at $14.4 \mathrm{~K}$, further confirming that AFM ground state stems from a FM-like immediate state.

Figures 3(a) and 3(b) exhibit the isothermal magnetization with field up to $50 \mathrm{kOe}$ applied along in-plane and out-of-plane directions, respectively, from $2 \mathrm{~K}$ to 30 $\mathrm{K}$ with a temperature step of $1 \mathrm{~K}$. At high temperature, the curves are almost linear, suggesting a PM behavior. With decreasing temperature, the curves bend with negative curvatures, indicating an FM interaction. At low temperature, there is a rapid linear increase at low field and the magnetic moment is fully polarized in the highfield region. Based on the classical thermodynamical and the Maxwell's thermodynamic relation, the magnetic entropy change $\Delta S_{M}(T, H)$ is given by:24,25

$$
\Delta S_{M}=\int_{0}^{H}\left[\frac{\partial S(T, H)}{\partial H}\right]_{T} d H=\int_{0}^{H}\left[\frac{\partial M(T, H)}{\partial T}\right]_{H} d H,
$$

where $[\partial S(T, H) / \partial H]_{T}=[\partial M(T, H) \partial T]_{H}$ is based on the Maxwell's relation. For magnetization measured at small temperature and field intervals,

$$
\Delta S_{M}=\frac{\int_{0}^{H} M\left(T_{i+1}, H\right) d H-\int_{0}^{H} M\left(T_{i}, H\right) d H}{T_{i+1}-T_{i}} .
$$

The calculated $-\Delta S_{M}(T, H)$ are presented in Figs. 3(a) and $3(\mathrm{~b})$. All the curves exhibit a peak feature, and the peak broadens asymmetrically on both sides with increasing magnetic field. For both field directions, the peak position gradually shifts from $15 \mathrm{~K}$ for $2 \mathrm{kOe}$ to 19 $\mathrm{K}$ for $50 \mathrm{kOe}$. The $-\Delta S_{M}$ reaches to a maximum value
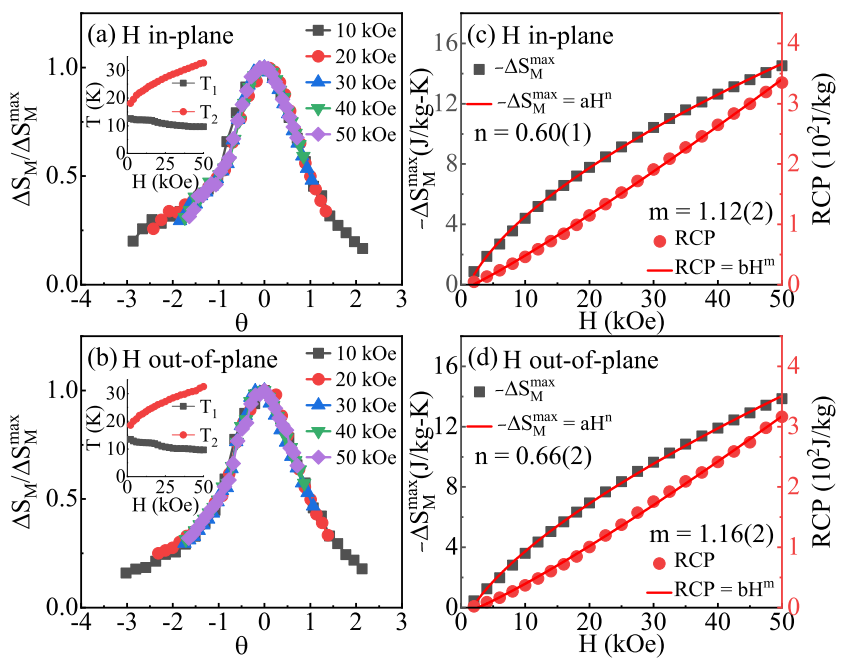

FIG. 4. (Color online). Normalized $\Delta S_{M} / \Delta S_{M}^{\max }$ as a function of the rescaled temperature $\theta$ for (a) in-plane and (b) out-of-plane fields. Insets show the evolution of the reference temperatures $T_{1}$ and $T_{2}$. Field dependence of the maximum magnetic entropy change $-\Delta S_{M}^{\max }$ and the relative cooling power RCP with power law fitting in red solid lines for (c) in-plane and (d) out-of-plane fields.

$\sim 14.6 \mathrm{~J} \mathrm{~kg}^{-1} \mathrm{~K}^{-1}$ for in-plane field and $13.8 \mathrm{~J} \mathrm{~kg}^{-1} \mathrm{~K}^{-1}$ for out-of-plane field, respectively.

There is a generalized magnetic entropy scaling analysis proposed for the second-order phase transition magnetocaloric materials $\frac{26}{2}$ In this approach the normalized magnetic entropy $\Delta S_{M} / \Delta S_{M}^{\max }$, estimated for each constant magnetic field, is scaled to the reduced temperature $\theta$ as defined in the following equations:

$$
\begin{aligned}
& \theta_{-}=\left(T_{\text {peak }}-T\right) /\left(T_{r 1}-T_{\text {peak }}\right), T<T_{\text {peak }}, \\
& \theta_{+}=\left(T-T_{\text {peak }}\right) /\left(T_{r 2}-T_{\text {peak }}\right), T>T_{\text {peak }},
\end{aligned}
$$

where $T_{r 1}$ and $T_{r 2}$ are the lower and upper temperatures at full-width half maximum of $\Delta S_{M} / \Delta S_{M}^{\max }$. In this method, $T_{c}$ fails to be a good parameter whereas $T_{\text {peak }}$ serves the purpose because of its field dependence. The normalized $\Delta S_{M} / \Delta S_{M}^{\max }$ roughly collapses on to a universal curve around $T_{\text {peak }}$ at indicated fields [Figs. 4(a) and 4(b)], indicating the feature of second-order PM-FM transition in $\mathrm{CrCl}_{3}$. Another parameter to characterize the potential magnetocaloric effect of materials is the relative cooling power (RCP): 27

$$
R C P=-\Delta S_{M}^{\max } \times \delta T_{F W H M},
$$

where the FWHM means the full width at half maximum of $-\Delta S_{M}$ curve. The RCP reaches a maximum value of $340.3 \mathrm{~J} \mathrm{~kg}^{-1}$ for in-plane field and $317.3 \mathrm{~J} \mathrm{~kg}^{-1}$ for out-ofplane field, respectively. In addition, the field dependence of $-\Delta S_{M}^{\max }$ and RCP can be well fitted by using the power-law relations $-\Delta S_{M}^{\max }=a H^{n}$ and $R C P=b H^{m}$ [Figs. 4(c) and 4(d)] $\stackrel{28}{2}$ The $-\Delta S_{M}^{\max }$ of $\mathrm{CrCl}_{3}$ is smaller 

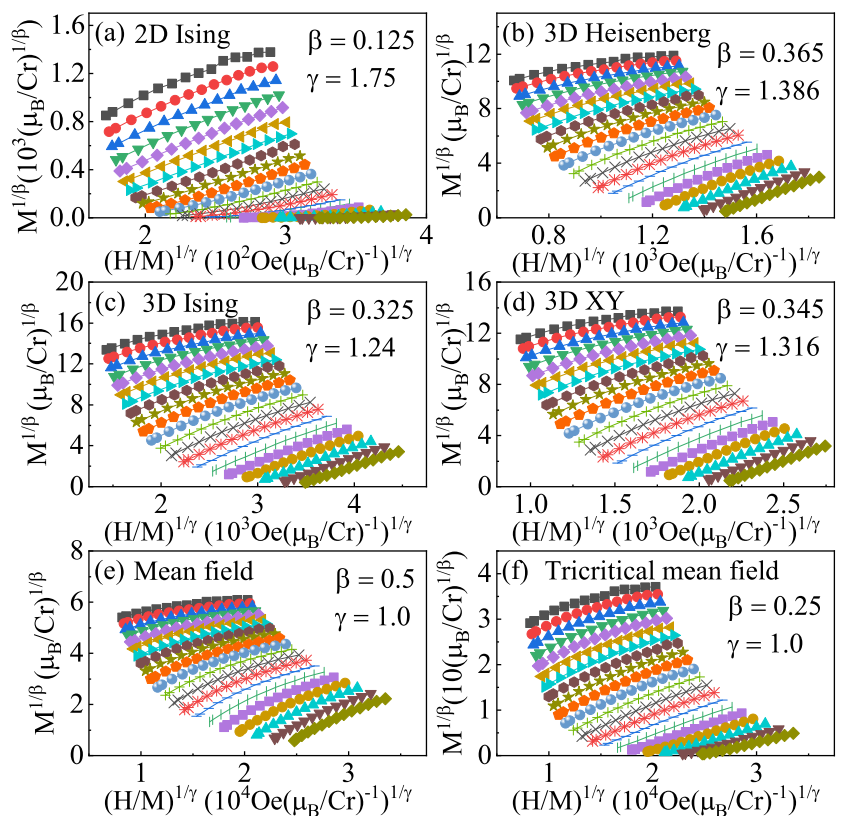

$(\mathrm{H} / \mathrm{M})^{1 / \gamma}\left(10^{3} \mathrm{Oe}\left(\mu_{\mathrm{B}} / \mathrm{Cr}\right)^{-1}\right)^{1 / \gamma}$
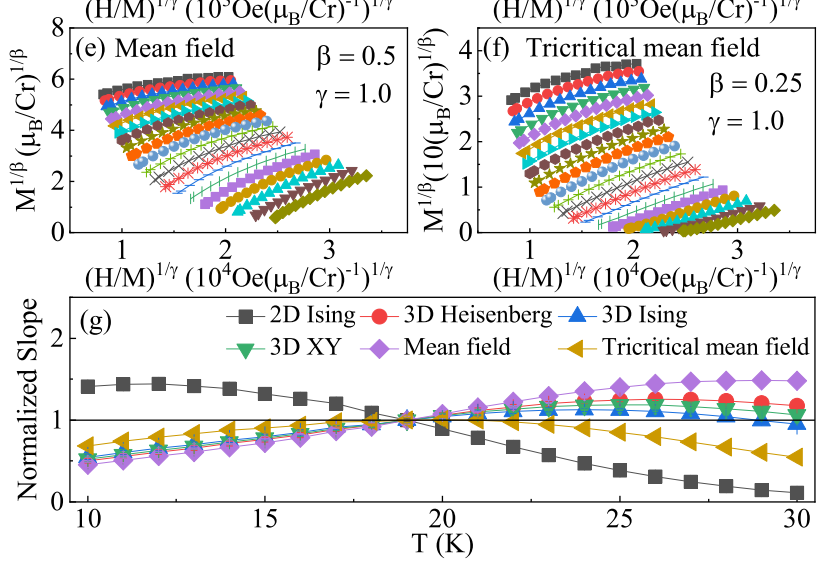

FIG. 5. (Color online). The modified Arrott plots $M^{1 / \beta}$ vs $(H / M)^{1 / \gamma}$ for in-plane fields with parameters of (a) $2 \mathrm{D}$ Ising, (b) 3D Heisenberg, (c) 3D Ising, (d) 3D XY, (e) Mean field, and (f) Tricritical mean field models. (g) Temperature dependence of the normalized slopes $N S=S(T) / S\left(T_{c}\right)$ for different theoretical models.

than that of well-known magnetic refrigerating materials with first-order transition, $\stackrel{29}{,}$ but is larger than some with second-order transition ${ }^{30-32}$ For instance, the $-\Delta S_{M}^{\max }$ of $\mathrm{R}_{6} \mathrm{Co}_{1.67} \mathrm{Si}_{3}(\mathrm{R}=\mathrm{Pr}, \mathrm{Gd}$, and $\mathrm{Tb})$ are $6.9,5.2$ and 7.0 $\mathrm{J} \mathrm{kg}^{-1} \mathrm{~K}^{-1}$ at $50 \mathrm{kOe}$, and for $\mathrm{CdCr}_{2} \mathrm{~S}_{4}$ is $7.0 \mathrm{~J} \mathrm{~kg}^{-1} \mathrm{~K}^{-1}$ at the same field 33,34 It is worth noting that the values of $-\Delta S_{M}^{\max }$ and $\mathrm{RCP}$ of $\mathrm{CrCl}_{3}$ are also larger than those of its cousin $\mathrm{CrBr}_{3}\left(7.2 \mathrm{~J} \mathrm{~kg}^{-1} \mathrm{~K}^{-1}\right.$ and $\left.191.5 \mathrm{~J} \mathrm{~kg}^{-1}\right)$ and $\mathrm{CrI}_{3}\left(4.24 \mathrm{~J} \mathrm{~kg}^{-1} \mathrm{~K}^{-1}\right.$ and $\left.122.6 \mathrm{~J} \mathrm{~kg}^{-1}\right), \underline{18.19}$ Thus, bulk $\mathrm{CrCl}_{3}$ could be a promising candidate for cryogenic magnetic refrigerating materials.

For a second-order PM-FM phase transition, the spontaneous magnetization $\left(M_{s}\right)$ below $T_{c}$, the initial magnetic susceptibility $\left(\chi_{0}^{-1}\right)$ above $T_{c}$, and the fielddependent magnetization (M) at $T_{c}$ are characterized by a set of critical exponents $\beta, \gamma$, and $\delta$, respectively $\underline{35}$ The mathematical definitions of the exponents from magnetization measurement are given below:

$$
\begin{gathered}
M_{s}(T)=M_{0}(-\varepsilon)^{\beta}, \varepsilon<0, T<T_{c}, \\
\chi_{0}^{-1}(T)=\left(h_{0} / m_{0}\right) \varepsilon^{\gamma}, \varepsilon>0, T>T_{c},
\end{gathered}
$$
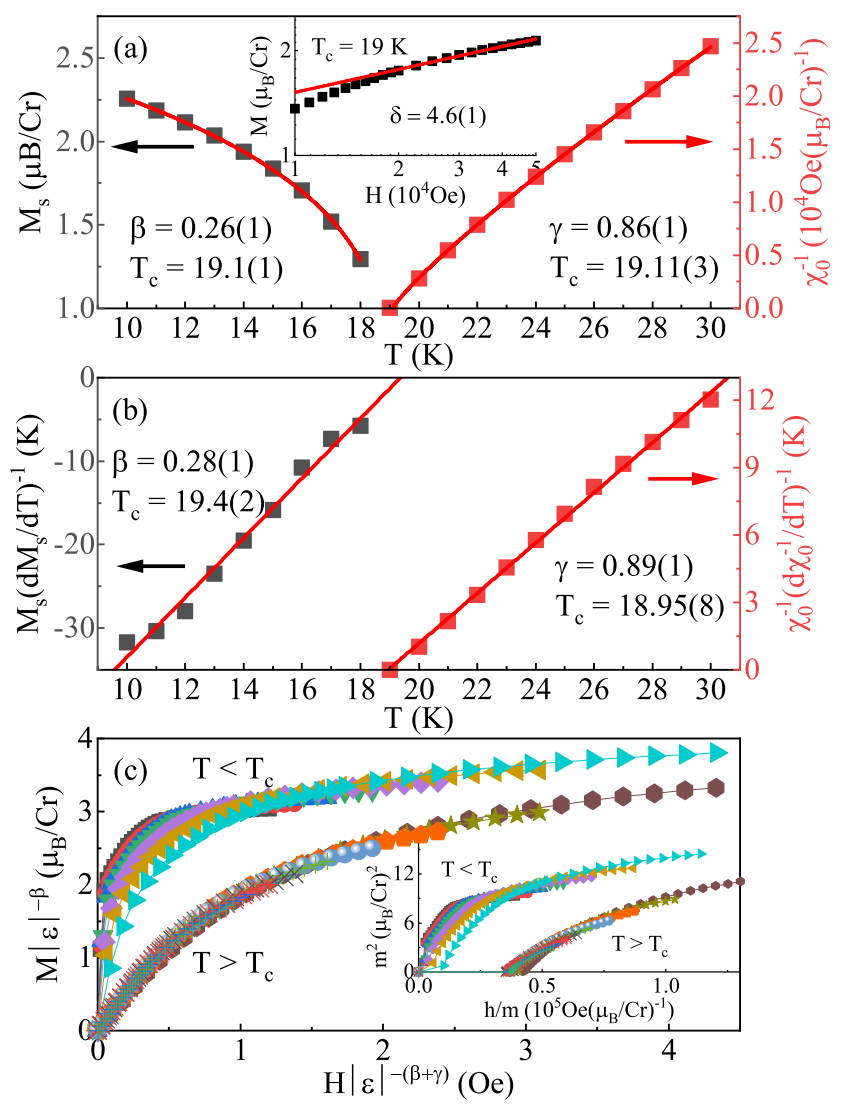

FIG. 6. (Color online). (a) Temperature dependence of the spontaneous magnetization $M_{s}$ (left) and the inverse initial susceptibility $\chi_{0}^{-1}$ (right) with solid fitting curves for $\mathrm{CrCl}_{3}$. Inset shows the $\log _{10} M$ vs $\log _{10} H$ at $T_{c}=19 \mathrm{~K}$ with linear fitting curve. (b) Kouvel-Fisher plots of $M_{s}\left(d M_{s} / d T\right)^{-1}$ (left) and $\chi_{0}^{-1}\left(d \chi_{0}^{-1} / d T\right)^{-1}$ (right) with solid fitting curves for $\mathrm{CrCl}_{3}$. (c) Scaling plots of renormalized $m=M|\varepsilon|^{-\beta}$ vs $h=H|\varepsilon|^{-(\beta+\gamma)}$ above and below $T_{c}$ for $\mathrm{CrCl}_{3}$. Inset shows the Arrott plot of $m^{2}$ vs $h / m$ for $\mathrm{CrCl}_{3}$.

$$
M=D H^{1 / \delta}, T=T_{c},
$$

where $\varepsilon=\left(T-T_{c}\right) / T_{c} ; M_{0}, h_{0} / m_{0}$ and $D$ are the critical amplitudes $\frac{36}{6}$ For the original Arrott plot, $\beta=0.5$ and $\gamma=1.0 \stackrel{37}{=}$ In a more general case with different critical exponents, the Arrott-Noaks equation of state provides a modified Arrott plot: ${ }^{38}$

$$
(H / M)^{1 / \gamma}=a \varepsilon+b M^{1 / \beta},
$$

where $\varepsilon=\left(T-T_{c}\right) / T_{c}$ and $a$ and $b$ are fitting constants. Figures 5(a)-5(f) present the modified Arrott plots for easy in-plane fields using theoretical critical exponents from 2D Ising $(\beta=0.125, \gamma=1.75), 3 \mathrm{D}$ Heisenberg $(\beta=$ $0.365, \gamma=1.386), 3 \mathrm{D}$ Ising $(\beta=0.325, \gamma=1.24), 3 \mathrm{D}$ $\mathrm{XY}(\beta=0.345, \gamma=1.316)$, mean-field $(\beta=0.5, \gamma=1.0)$ and tricritical mean-field $(\beta=0.25, \gamma=1.0)$ models $\underline{39}-\underline{4}$ There should be a set of parallel lines in the high fields with the same slope $S(T)=d M^{1 / \beta} / d(H / M)^{1 / \gamma}$. Comparing the normalized slope $\left[N S=S(T) / S\left(T_{c}\right)\right]$ with the 
ideal value of 1 enables us to determine the most suitable model, as shown in Fig. 5(g). It is clearly seen that the $N S$ of 2 D Ising model shows the largest deviation from 1. The $N S$ of tricritical mean field model is close to $N S=1$ mostly below $T_{c}$, while that of $3 \mathrm{D}$ Ising model is the best above $T_{c}$.

To generate the actual critical exponents of bulk $\mathrm{CrCl}_{3}$, the linearly extrapolated $M_{s}$ and $\chi_{0}^{-1}$ are plotted against temperature in Fig. 6(a) $\stackrel{42}{=}$ According to Eqs. (6) and (7), the solid fitting lines give that $\beta=0.26(1)$, with $T_{c}=$ 19.1(1) $\mathrm{K}$, and $\gamma=0.86(1)$, with $T_{c}=19.11(3) \mathrm{K}$. According to Eq. (8), the $M(H)$ at $T_{c}$ should be a straight line in log-log scale with the slope of $1 / \delta$. Such fitting yields $\delta=4.6(1)$ [inset in Fig. 6(a)], which agrees with the calculated $\delta=4.3(1)$ from the obtained $\beta$ and $\gamma$ based on the Widom relation $\delta=1+\gamma / \beta$ ! $\underline{43}$ The more accurate values of critical exponents could be obtained using the Kouvel-Fisher technique, where $M_{s}(T) /\left(d M_{s}(T) / d T\right)^{-1}$ and $\chi_{0}^{-1}(T) /\left(d \chi_{0}^{-1}(T) / d T\right)^{-1}$ plotted against temperature should be straight lines with slopes $1 / \beta$ and $1 / \gamma$, respectively $\underline{44}$ The linear fits to the plots [Fig. 6(b)] yield the values of critical exponents and $T_{c}$ are $\beta=0.28(1)$, with $T_{c}=19.4(2) \mathrm{K}$, and $\gamma=0.89(1)$, with $T_{c}=18.95(8)$ $\mathrm{K}$. The value of $\beta$ for a $2 \mathrm{D}$ magnet should be within a window $0.1 \leq \beta \leq 0.25 \stackrel{45}{\underline{4}}$ suggesting a possible $3 \mathrm{D}$ magnetic behavior of $\mathrm{CrCl}_{3}$. The obtained values of critical exponents are close to those of theoretical tricritical mean field model $(\beta=0.25$ and $\gamma=1.0)$, indicating that the second-order PM-FM transition is situated close to a 3D to $2 \mathrm{D}$ critical point.

Scaling analysis can be used to estimate the reliability of the obtained critical exponents. According to scaling hypothesis, the magnetic equation of state in the critical region obeys a scaling relation can be expressed as:

$$
M(H, \varepsilon)=\varepsilon^{\beta} f_{ \pm}\left(H / \varepsilon^{\beta+\gamma}\right),
$$

where $f_{+}$for $T>T_{c}$ and $f_{-}$for $T<T_{c}$, respectively, are the regular functions. In terms of the variable $m \equiv \varepsilon^{-\beta} M(H, \varepsilon)$ and $h \equiv \varepsilon^{-(\beta+\gamma)} H$, scaled or renormalized magnetization and scaled or renormalized field, respectively, Eq.(10) reduces to a simple form:

$$
m=f_{ \pm}(h) .
$$

It implies that for a true scaling relation with proper selection of $\beta, \gamma$, and $\delta$, the scaled $m$ versus $h$ data will fall onto two different universal curves; $f_{+}(h)$ for temperature above $T_{c}$ and $f_{-}(h)$ for temperature below $T_{c}$. Using the values of $\beta$ and $\gamma$ obtained from the KouvelFisher plot, we have constructed the scaled $m$ vs scaled $h$ plot in Fig. 6(c). It is clear from the plots that all the experimental data collapse onto two different branches: one above $T_{c}$ and another below $T_{c}$. The deviation at low fields below $T_{c}$ probably arise from the spin dynamics behavior of $\mathrm{CrCl}_{3}$. The scaling analysis can be also verified from plots of $m^{2}$ vs $h / m$ [inset in Fig. 6(c)], confirming proper treatment of the critical regime.

\section{CONCLUSIONS}

In summary, we have studied in details the magnetism and magnetocaloric effect of bulk $\mathrm{CrCl}_{3}$ single crystal. The two-step magnetic transition at $T_{N}=14.4 \mathrm{~K}$ and $T_{c}$ $=16 \mathrm{~K}$ was clearly characterized by low-field dc and ac magnetization measurements. Further neutron scattering measurement is of interest to shed more light on its microscopic mechanism. The magnetic entropy change $-\Delta S_{M}^{\max } \sim 14.6 \mathrm{~J} \mathrm{~kg}^{-1} \mathrm{~K}^{-1}$ and the relative cooling power $R C P \sim 340.3 \mathrm{~J} \mathrm{~kg}^{-1}$ with in-plane field change of $50 \mathrm{kOe}$ indicates that $\mathrm{CrCl}_{3}$ would be a promising candidate material for cryomagnetic refrigeration. The second-order in nature of the PM-FM transition near $T_{c}$ has been verified by the scaling analysis of $-\Delta S_{M}$. A set of critical exponents $\beta, \gamma$, and $\delta$ estimated from various techniques match reasonably well and follow the scaling equation, indicating that the PM-FM transition of bulk $\mathrm{CrCl}_{3}$ is situated close to a $3 \mathrm{D}$ to $2 \mathrm{D}$ critical point. Considering its magnetism can be maintained upon exfoliating bulk crystal down to a monolayer,$\underline{46}-\underline{-51}$ further study on the size-dependent properties is of interest.

\section{ACKNOWLEDGEMENTS}

This work was supported by the US DOE-BES, Division of Materials Science and Engineering, under Contract No. de-sc0012704 (BNL).
1 B. Huang, G. Clark, E. Navarro-Moratalla, D. R. Klein, R. Cheng, K. L. Seyler, D. Zhong, E. Schmidgall, M. A. McGuire, D. H. Cobden, W. Yao, D. Xiao, P. JarilloHerrero, and X. D. Xu, Nature 546, 270 (2017).

2 M. A. McGuire, G. Clark, S. KC, W. M. Chance, G. E. Jellison, Jr., V. R. Cooper, X. Xu, and B. C. Sales, Phys. Rev. M 1014001 (2017).

${ }^{3}$ K. L. Seyler, D. Zhong, D. R. Klein, S. Guo, X. Zhang, B. Huang, E. Navarro-Moratalla, L. Yang, D. H. Cobden, M. A. McGuire, W. Yao, D. Xiao, P. Jarillo-Herrero, and X. D. Xu, Nat. Phys. 14, 277 (2018).
${ }^{4}$ C. Gong, L. Li, Z. L. Li, H. W. Ji, A. Stern, Y. Xia, T. Cao, W. Bao, C. Z. Wang, Y. Wang, Z. Q. Qiu, R. J. Cava, S. G. Louie, J. Xia, and X. Zhang, Nature 546, 265 (2017).

5 B. Huang, J. Cenker, X. Zhang, E. L. Ray, T. Song, T. Taniguchi, K. Watanabe, M. A. McGuire, D. Xiao, and X. D. Xu, Nat. Nanotech. 15, 212 (2020).

6 B. Huang, G. Clark, D. R. Klein, D. MacNeill, E. NavarroMoratalla, K. L. Seyler, N. Wilson, M. A. McGuire, D. H. Cobden, D. Xiao, W. Yao, P. Jarillo-Herrero, and X. D. $\mathrm{Xu}$, Nat. Nanotech. 13, 544 (2018).

7 S. Jiang, L. Li, Z. Wang, K. F. Mak, and J. Shan, Nat. Nanotech. 13, 549 (2018). 
8 X. Cai, T. Song, N. P. Wilson, G. Clark, M. He, X. Zhang, T. Taniguchi, K. Watanabe, W. Yao, D. Xiao, M. A. McGuire, D. H. Cobden, and X. Xu, Nano Lett. 19, 3993 (2019).

9 M. A. McGuire, H. Dixit, V. R. Cooper, and B. C. Sales, Chem. Mater. 27, 612 (2015).

10 J. F. Dillon and C. E. Olson, J. Appl. Phys. 36, 1259 (1965).

11 I. Tsubokawa, J. Phys. Soc. Jpn. 15, 1664 (1960).

12 J. W. Cable, M. K. Wilkinson, and E. O. Wollan, J. Phys. Chem. Solids 19, 29 (1961).

13 W. N. Hansen and M. Griffel, J. Chem. Phys. 28, 902 (1958).

14 C. Starr, F. Bitter, and A. Kaufmann, Phys. Rev. 58, 977 (1940).

15 B. Kuhlow, Phys. Status Solidi 72, 161 (1982).

16 H. Bizette, A. Adam, and C. Terrier, C. R. Acad. Sci. 252, 1571 (1961).

17 N. Bykovetz, A. Hoser, and C. L. Lin, AIP Adv. 9, 035029 (2019).

18 Y. Liu and C. Petrovic, Phys. Rev. B 97, 174418 (2018).

19 X. Yu, X. Zhang, Q. Shi, S. Tian, H. Lei, K. Xu, and H. Hosono, Front. Phys. 14, 43501 (2019).

20 Y. Liu and C. Petrovic, Phys. Rev. B 97, 014420 (2018).

${ }^{21}$ G. T. Lin, X. Luo, F. C. Chen, J. Yan, J. J. Gao, Y. Sun, W. Tong, P. Tong, W. J. Lu, Z. G. Sheng, W. H. Song, X. B. Zhu, and Y. P. Sun, Appl. Phys. Lett. 112, 072405 (2018).

22 L. L. Handy and N. W. Gregory, J. Am. Chem. Soc, 74, 891 (1952).

23 M. Abramchuk, S. Jaszewski, K. R. Metz, G. B. Osterhoudt, Y. Wang, K. S. Burch, and F. Tafti, Adv. Mater. 30, 1801325 (2018).

24 V. Pecharsky, K. Gscheidner, J. Magn. Magn. Mater. 200, 44 (1999).

25 J. Amaral, M. Reis, V. Amaral, T. Mendonc, J. Araujo, M. Sa, P. Tavares, J. Vieira, J. Magn. Magn. Mater. 290, 686 (2005).

${ }^{26}$ V. Franco and A. Conde, Int. J. Refrig. 33, 465 (2010).

27 K. A. Gschneidner Jr., V. K. Pecharsky, A. O. Pecharsky, and C. B. Zimm, Mater. Sci. Forum 315, 69 (1999).

28 V. Franco, J. S. Blazquez, and A. Conde, Appl. Phys. Lett. 89, 222512 (2006).

29 K. A. Gschneidner Jr., V. K. Pecharsky, and A. O. Tsokol, Rep. Prog. Phys. 68, 1479 (2005).
30 M.-H. Phan and S.-C. Yu, J. Magn. Magn. Mater. 308, 325 (2007).

31 N. S. Bingham, M.-H. Phan, H. Srikanth, M. A. Torija, and C. Leighton, J. Appl. Phys. 106, 023909 (2009).

32 A. G. Gamzatov, Y. S. Koshkudko, D. C. Freitas, E. Moshkina, L. Bezmaternykh, A. M. Aliev, S.-C. Yu, and M.-H. Phan, arXiv:2005.04572,

33 B. G. Shen, J. R. Sun, F. X. Hu, H. W. Zhang, and Z. H. Cheng, Adv. Mater. 21, 4545 (2009).

34 A. M. Tishin, Y. I. Spichkin, The Magnetocaloric Effect and its Applications (Institute of Physics Publishing, Bristol, UK, 2003).

35 H. E. Stanley, Introduction to Phase Transitions and Critical Phenomena (Oxford U. P., London and New York, 1971).

36 M. E. Fisher, Rep. Prog. Phys. 30, 615 (1967).

37 A. Arrott, Phys. Rev. B 108, 1394 (1957).

38 A. Arrott, and J. Noakes, Phys. Rev. Lett. 19, 786 (1967).

39 S. N. Kaul, J. Magn. Magn. Mater. 53, 5 (1985).

40 K. Huang, Statistical Machanics, 2nd ed. (Wiley, New York, 1987).

41 J. C. LeGuillou, and J. Zinn-Justin, Phys. Rev. B 21, 3976 (1980).

42 A. K. Pramanik, and A. Banerjee, Phys. Rev. B 79, 214426 (2009).

43 B. Widom, J. Chem. Phys. 41, 1633 (1964).

44 J. S. Kouvel, and M. E. Fisher, Phys. Rev. 136, A1626 (1964).

45 A. Taroni, S. T. Bramwell, and P. C. W. Holdsworth, J. Phys.: Condens. Matter 20, 275233 (2008).

46 O. Besbes, S. Nikolaev, N. Meskini, and I. Solovyev, Phys. Rev. B 99, 104432 (2019).

47 F. Xue, Y. Hou, Z. Wang, and R. Wu, Phys. Rev. B 100, 224429 (2019).

48 D. MacNeill, J. T. Hou, D. R. Klein, P. Zhang, P. JarilloHerrero, and L. Liu, Phys. Rev. Lett. 123, 047204 (2019).

49 C. A. Pocs, I. A. Leahy, H. Zheng, G. Cao, E. S. Choi, S. H. Do, K. Y. Choi, B. Normand, and M. Lee, Phys. Rev. Research 2, 013059 (2020).

50 S. Kazim, M. Ali, S. Palleschi, G. D'Olimpio, D. Mastrippolito, A. Politano, R. Gunnella, A. D. Cicco, M. Renzelli, and G. Moccia, Nanotechnology in press https://doi.org/10.1088/1361-6528/ab7de6.

51 X. Lu, R. Fei, and L. Yang, arXiv:2002.05208v1. 